\title{
HÜCKEL AND MÖBIUS ANNULENES: RELATIONSHIPS BETWEEN THEIR HÜCKEL-LEVEL ORBITALS AND THEIR HÜCKEL-LEVEL ENERGIES
}

\author{
Richard Francis Langler \\ Department of Chemistry, Mount Allison University, Sackville, New Brunswick, Canada E4L 1G8 \\ Guillermo Salgado and Carlos Mendizabal \\ Universidad Santo Tomas, Agropecuaria, Av. Ejercito 146, Santiago, Chile
}

Recebido em 28/10/99; aceito em 29/2/00

\begin{abstract}
The problem of convenient access to quantitative Hückel-level descriptions of Möbius and Hückel annulenes for undergraduate lectures about aromaticity is discussed. Frost circle, Zimmerman circle, double circle and Langler semicircular mnemonics are described. The relationship between spectra (complete sets of secular equation roots) for an isoconjugate pair of Hückel and Möbius annulenes and the corresponding acyclic polyene with one less carbon is fully developed. In addition to providing an alternative path to exact spectrum roots, this relationship provides immediate access to almost half of the eigenfunctions for an isoconjugate annulene pair. The remaining eigenfunctions may be obtained very easily.
\end{abstract}

Keywords: aromaticity; circle mnemonics; Hückel theory.

\section{INTRODUCTION}

In 1931, Hückel described a very simple molecular orbital approach to the $\pi$-electronic structure of planar molecules ${ }^{1}$. Since then, many textbooks have been written which introduce Hückel theory to undergraduate students and apply it, primarily, to planar hydrocarbons (see reference 2 for some representative texts). Furthermore, many concerted chemical reactions (e.g. Diels-Alder) can be understood using either the original Zimmerman-Dewar method ${ }^{3}$ or a modification of $i^{4}$, both of which rely directly on Hückel theory.

The manual application of Hückel theory to a hydrocarbon with $\mathrm{n}$ carbon atoms requires one to (i) write a set of $\mathrm{n}$ homogeneous linear equations, (ii) generate an $\mathrm{n} \times \mathrm{n}$ secular determinant, (iii) diagonalize the determinant to generate an nth order polynomial (the secular equation), (iv) obtain $\mathrm{n}$ roots for the secular equation (the set of n roots is called the "spectrum" of the compound $\left.{ }^{5}\right)$, (v) calculate the $n$ molecular orbital energies (the eigenvalues) using the numbers in the spectrum of the compound and (vi) obtain n molecular orbitals (eigen-functions) for the molecule by substituting the spectrum roots back into the homogeneous linear equations.

Because the foregoing approach is very tedious, two alternatives have emerged: (A) exact Hückel-level generalizations have been developed for particular classes of molecules and (B) computer programs have been written to do this work quickly. We note that MATLAB ${ }^{6}$ is a particularly robust and readily available FORTRAN program for handling determinants.

Amongst hydrocarbons, the annulenes are particularly interesting because of the special properties associated with both aromatic and antiaromatic molecules. Moreover, aromatic/ antiaromatic annulenes are directly invoked in applying the previously mentioned methods $\mathrm{s}^{3,4}$ for understanding concerted reactions. Hence, the annulenes are much discussed in undergraduate lectures and have been selected as the centerpiece for this article.

Two classes of annulenes have been subjected to examination at the Hückel level - (i) planar structures with parallel p-orbitals (Hückel annulenes) and, following Heilbronner ${ }^{7}$, Möbius annulenes in which each p-orbital has been rotated by $\Theta^{\circ}$ relative to its contiguous neighbours. Figure 1 shows the p-orbital arrays for both Huckel and Möbius cyclobutadiene.

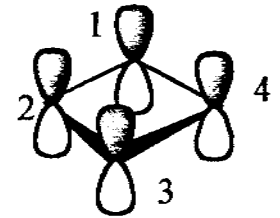

Hückel cyclobutadiene

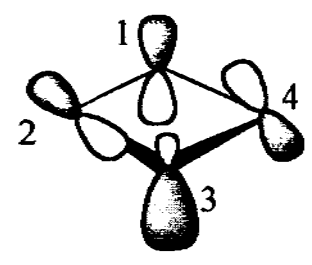

Möbius cyclobutadiene
Figure 1.

Because qualitative discussions about aromaticity for Hückel and Möbius annulenes focus on ground state multiplicity, some of the simple circle mnemonics, described below, are normally included in undergraduate lectures. Therefore, the current report will explore convenient rapid methods to obtain exact Hückellevel spectra and eigenfunctions from these mnemonics.

\section{RESULTS AND DISCUSSION}

\section{(A) Hückel Spectra and Orbital Energies (Eigenvalues)}

\section{(i) The Frost and Zimmerman Circle Mnemonics}

In 1953, Frost and Musulin ${ }^{8}$ pointed out that each contact point for a regular polygon inscribed in a circle (polygon has a vertex down) corresponds to the relative energy for a molecular orbital of the corresponding Hückel annulene. Figure 2 illustrates the Frost circle mnemonic for Hückel cyclopropenyl. Since the eigenvalues for the molecular orbitals are obtained from the spectrum of the molecule in question, the next problem is to obtain the spectrum for any Huickel annulene without doing full Hückel calculations. Salgado Moran showed ${ }^{9}$ that simple trigonometry will suffice to obtain the spectrum for Hückel annulenes inscribed in a circle with a two unit radius (a representative spectrum is provided for Hückel cyclopropenyl in Figure 2).

In 1966, Zimmerman ${ }^{10}$ revealed the corresponding circle mnemonic (polygon has a side down) for Möbius annulenes. Figure 3 illustrates the Zimmerman circle mnemonic for Möbius cyclopropenyl. Salgado Moran's approach using simple trigonometry will provide the spectrum for a Möbius annulene too (see Figure 4 for an example). 


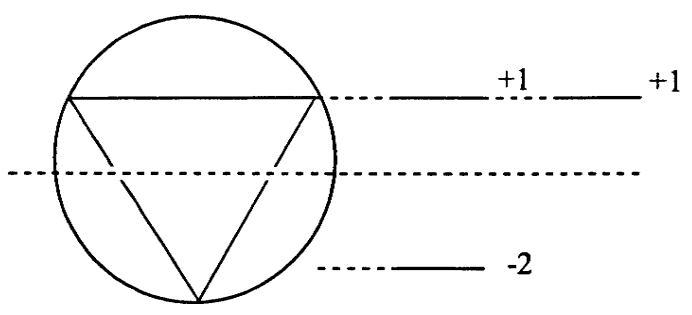

Figure 2. Frost circle mnemonic for Hückel cyclopropenyl. The polygon is inscribed in a circle with the vertex down. Each point at which a polygon vertex touches the circle circumference corresponds to the relative energy of a Hïckel cyclopropenyl molecular orbital.

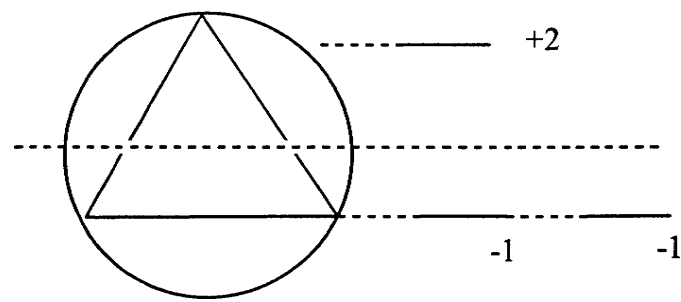

Figure 3. Zimmerman circle mnemonic for Möbius cyclopropenyl. The polygon is inscribed in a circle with a side down. Each point at which a polygon vertex touches the circle circumference corresponds to the relative energy of a Möbius cyclopropenyl molecular orbital.

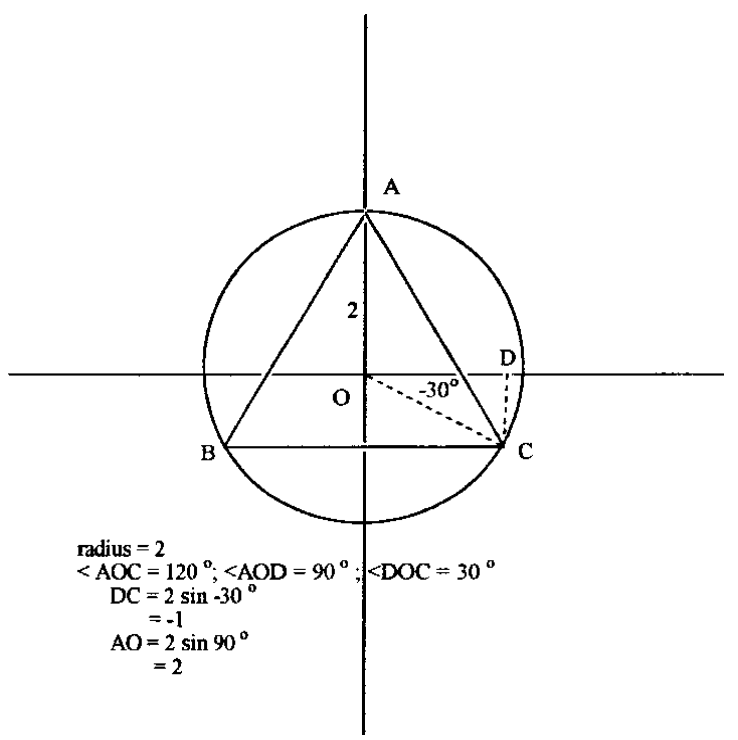

Figure 4. Simple trigonometry in conjunction with the Zimmerman circle mnemonic for Möbius cyclopropenyl.

Each polynomial root $\left(r_{1}, r_{2}, r_{3} \ldots r_{i}\right)$ in the spectrum of a Hückel annulene can be converted into the desired eigenvalue using the expression in equation (1).

$\mathrm{E}_{\mathrm{i}}^{\mathrm{H}}=\alpha-\mathrm{r}_{\mathrm{i}} \beta^{\mathrm{H}}$

Thus, Hückel cyclopropenyl has the eigenvalues: ${ }^{\wedge} \alpha+2 \beta^{\mathrm{H}}$, $\alpha-\beta^{\mathrm{H}}$ and $\alpha-\beta^{\mathrm{H}}$ (see spectrum, Figure 2 ).

Because the p-orbitals in a Möbius array are not supposed to be parallel, Heilbronner ${ }^{7}$ suggested that the spectrum would give rise to Möbius eigenvalues expressed in terms of a Möbius beta: $\beta^{\mathrm{M}}$ (see equation (2)).

$\mathrm{E}_{\mathrm{i}}^{\mathrm{M}}=\alpha-\mathrm{r}_{\mathrm{i}} \beta^{\mathrm{M}}$

In order to convert Möbius eigenvalues into a more familiar form, Heilbronner proposed that $r_{i} \beta^{M}$ should be multiplied by $\cos \Theta$. $\Theta$ s defined in equation (3), where $\mathrm{k}$ is the number of vertices in the annulene.

$\Theta=180 / \mathrm{k}$

Thus for Möbius cyclopropenyl, $\Theta=60^{\circ}, \cos \Theta=0.5$ and the eigenvalues are $\alpha+\beta^{\mathrm{M}}$ (or $\alpha+0.5 \beta^{\mathrm{H}}$ ), $\alpha+\beta^{\mathrm{M}}$ (or $\alpha+0.5 \beta^{\mathrm{H}}$ ) and $\alpha-2 \beta^{\mathrm{M}}$ (or $\alpha-\beta^{\mathrm{H}}$ ) (see Figure 3 for the spectrum of Möbius cyclopropenyl).

\section{(ii) Relating Hückel and Möbius Spectra: \\ A Double Circle Mnemonic}

Both Hückel and Möbius p-orbital arrays possess a $\mathrm{C}_{2}$ axis (there is, for example, a $C_{2}$ axis through vertices 1 and 3 in each of the cyclobutadienes shown in Figure 1). For each type of array, $\mathrm{p}_{3}$ must vanish in some orbitals. For Hückel cyclobutadiene, $\mathrm{p}_{3}$ vanishes from orbitals which require it to be symmetric with respect to rotation about the $\mathrm{C}_{2}$ axis through vertices 1 and 3 (hereafter referred to as "the $C_{2}$ axis"). For Möbius cyclobutadiene, $\mathrm{p}_{3}$ vanishes from molecular orbitals which require it to be antisymmetric with respect to rotation about the $\mathrm{C}_{2}$ axis. Thus, each structure will have some molecular orbitals with a maximum of 3 non-zero contiguous coefficients. These are the eigenfunctions of the allyl group and they are associated with the allyl spectrum. In general, isoconjugate Hückel and Möbius annulenes share the spectrum of the acyclic polyene which has one less carbon.

Since Hückel annulenes have a zero coefficient for one center in each of their symmetric (rotation about $\mathrm{C}_{2}$ ) orbitals, the spectrum of a Hückel annulene has those roots associated with the symmetric molecular orbitals of the acyclic polyene with one less carbon (i.e. $\psi_{2}, \psi_{4}, \psi_{6}, \ldots . \psi_{2 n+2}$ ). Since Möbius annulenes have a zero coefficient for one center in each of their antisymmetric (rotation about $\mathrm{C}_{2}$ ) orbitals, the spectrum of a Möbius annulene has those roots associated with the antisymmetric molecular orbitals of the acyclic polyene with one less carbon (i.e. $\psi_{1}, \psi_{3}, \psi_{5} \ldots \psi_{2 n+1}$ ). Figure 5 presents these relationships for the cyclobutadienes and the cyclopentadienyls.

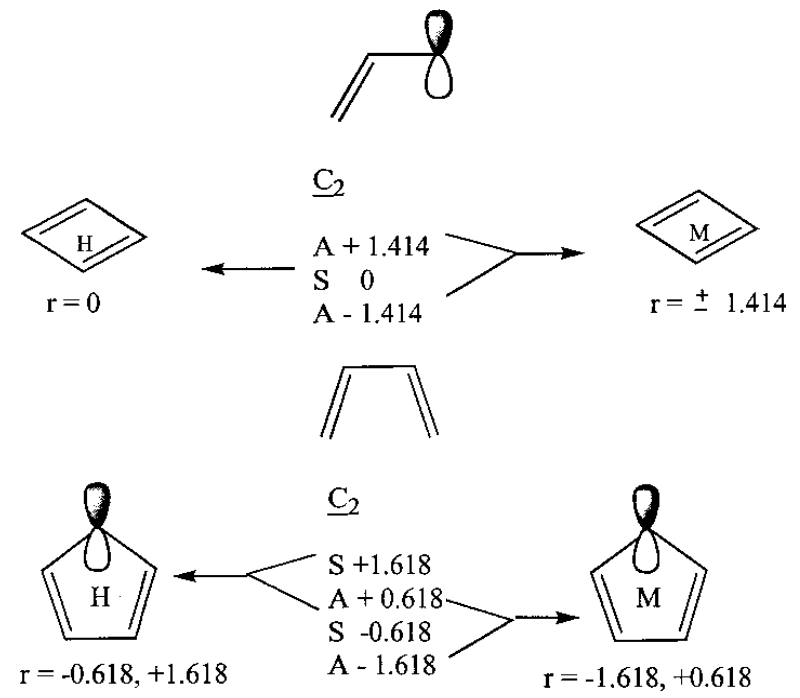

Figure 5. Deducing partial spectra for Hückel and Möbius annulenes given the spectrum of the acyclic polyene with one less carbon.

Given the Zimmerman and Frost circle mnemonics, along with the spectrum of the appropriate acyclic polyene, all secular equation roots are immediately known for any isoconjugate pair of Hückel and Möbius annulenes except those at \pm 2 . All 
even Hückel annulenes have roots at \pm 2 , while no even Möbius annulenes have such roots. All odd Hückel annulenes have a single root at -2 , while all odd Möbius annulenes have a single root at +2 . Consequently, the roots in the spectrum of the appropriate acyclic polyene will occur in degenerate pairs, along with roots at \pm 2 , in the sum of the spectra for any isoconjugate pair of Hückel and Möbius annulenes, regardless of the number of vertices in the annulene.

Each even Hückel annulene possesses a rotational axis which passes through diametrically opposed vertices. All molecular orbitals which are symmetric with respect to rotation about such an axis must have zero coefficients for the p-orbitals associated with the pair of centers the axis passes through. As a result, each $\mathrm{C}_{2 \mathrm{n}+2}$ Hückel annulene ( $\mathrm{C}_{6}$ or larger) has a subset of molecular orbitals which can be represented as simple linear combinations of the molecular orbitals for the corresponding $\mathrm{C}_{\mathrm{n}}$ acyclic polyene (e.g. cyclooctatetraene has three symmetric molecular orbitals, each of which can be represented as a linear combination of a degenerate pair of allyl molecular orbitals). These orbitals are associated with spectrum roots that are identical to those for the $C_{n}$ polyene.

From the Frost circle mnemonic, these roots will occur as degenerate pairs in the spectrum of the Hückel annulene. From the previous discussion, the Hückel annulene will also have spectrum roots at \pm 2 . Moreover, all of these roots will be present in the combined spectra of the $\mathrm{C}_{\mathrm{n}+1}$ Hückel and Möbius annulenes.

It is now obvious that one can inscribe both a $\mathrm{C}_{\mathrm{n}+1}$ polygon (vertex down) and a $\mathrm{C}_{n+1}$ polygon (side down) in the same circle and know that each contact point corresponds to the relative energy of a molecular orbital for the Hückel $C_{2 n+2}$ annulene. Figure 6 illustrates this "double circle mnemonic" for Hückel cyclooctatetraene. The double circle mnemonic illustrated in Figure 6 has been proposed earlier ${ }^{11}$ with only a very modest discussion of its origin.

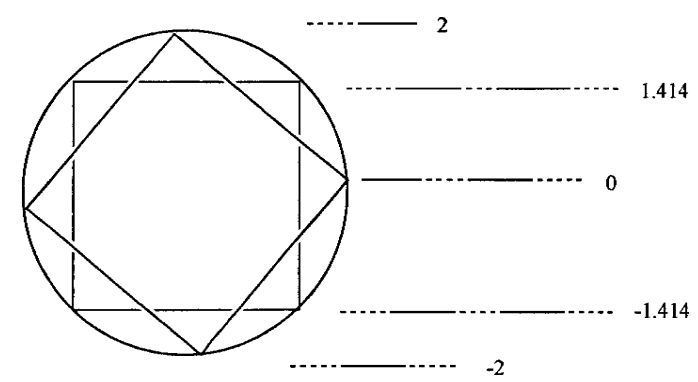

Figure 6. Obtaining the spectrum of Hückel cyclooctatetraene by inscribing Möbius and Hückel cyclobutadienes in a single circle.

\section{(iii) Beyond the Annulenes: A Semicircular Mnemonic for} Contiguous, Symmetric, Non-fused Bicycles

The Frost circle mnemonic (see section (A)(i) and Figure 2) provides a simple and convenient classroom approach to the generalization that Hückel annulenes are aromatic if they possess $4 n+2 \pi$ electrons and antiaromatic if they possess $4 n \pi$ electrons. A recently reported semicircular mnemonic ${ }^{12}$ provides rapid access to the relative energies of the molecular orbitals for Hückel contiguous, symmetrical, non-fused bicycles. Beginning with the application of the Frost circle mnemonic to the monocycle, one can extrapolate to obtain the relative energies for the orbitals of the Hückel bicycle. Figure 7 illustrates the application of this mnemonic device to biscyclobutadiene.

What emerged from the systematic exploitation of this mnemonic for symmetric, contiguous, non-fused bicycles is that (at the Hückel level) they are aromatic if they possess $8 n+2 \pi$ electrons and antiaromatic if they possess $8 \mathrm{n} \pi$ electrons. The foregoing generalization serves to establish that the widespread practice of applying Hückel's rule to polycycles is unsound.

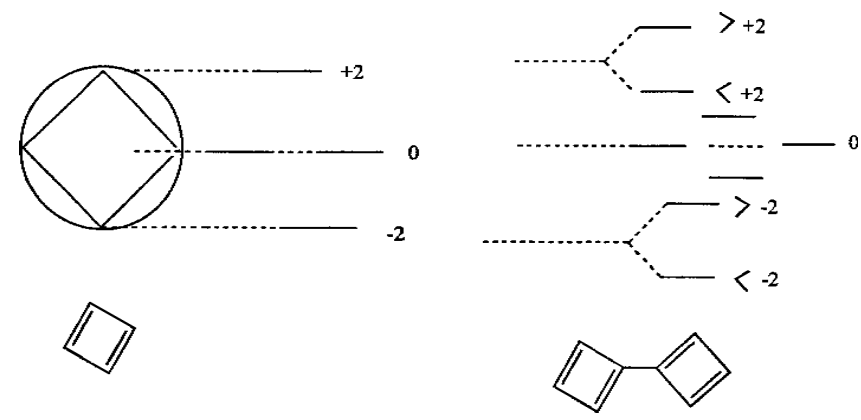

Figure 7. The Langler semicircular mnemonic applied to biscyclobutadiene.

\section{(B) Molecular Orbitals (Eigenfunctions)}

As outlined in the introduction, one begins to apply Hückel theory to a hydrocarbon by writing the appropriate homogeneous linear equations. After making the customary zeroth order Hückel assumptions, the homogeneous linear equations for Hückel cyclobutadiene are:
$\mathrm{a}_{1} \mathrm{r}+\mathrm{a}_{2}+0+\mathrm{a}_{4}=0$
$\mathrm{a}_{1}+\mathrm{a}_{2} \mathrm{r}+\mathrm{a}_{3}+0=0$
$0+\mathrm{a}_{2}+\mathrm{a}_{3} \mathrm{r}+\mathrm{a}_{4}=0$
$\mathrm{a}_{1}+0+\mathrm{a}_{3}+\mathrm{a}_{4} \mathrm{r}=0$

From Figure 5, Hückel cyclobutadiene has a symmetric molecular orbital for which $\mathrm{r}=0$. Its eigenfunction is identical to $\psi_{2}$ for the allyl group (see Figure 8 ). Because it has another molecular orbital for which $r=0$ (Frost circle mnemonic), that orbital must be antisymmetric. Therefore $a_{1}=a_{3}$ and, from equation (5), $\mathrm{a}_{1}=\mathrm{a}_{3}=0$. So, from equation (6), $\mathrm{a}_{2}=-\mathrm{a}_{4}$. Normalization leads to $\psi_{\mathrm{A}}$ (see Figure 8). Clearly, degenerate pairs of orbitals can be rapidly obtained for Hückel annulenes. For all Hückel annulenes, the absolute value of the coefficients in the orbitals for which $r= \pm 2$ can be obtained from equation $(8)$, where $\mathrm{k}$ is the number of vertices in the annulene.

$\mathrm{a}=\mathrm{k}^{-0.5}$

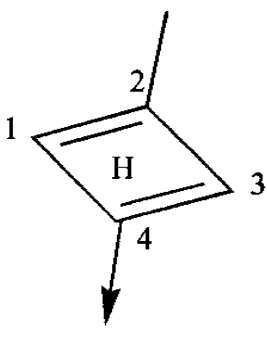

$\mathrm{C}_{2}$

$\mathbf{r}=0 ; \psi_{\mathrm{S}}: 0.707 \mathrm{p}_{1}-0.707 \mathrm{p}_{3}$

$\mathrm{r}=0 ; \psi_{\mathrm{A}}: 0.707 \mathrm{p}_{2}-0.707 \mathrm{p}_{4}$

Figure 8. Rapid access to degenerate eigenfunctions for Huckel cyclobutadiene.

For Hückel annulenes, $\mathrm{r}=-2$ orbitals have the form $\mathrm{p}_{1} \mathrm{k}^{-0.5}+$ $\mathrm{p}_{2} \mathrm{k}^{-0.5}+\ldots . .+\mathrm{p}_{\mathrm{n}} \mathrm{k}^{-0.5}$, whereas $\mathrm{r}=2$ orbitals have the form $\mathrm{p}_{1} \mathrm{k}^{-0.5}$ $-\mathrm{p}_{2} \mathrm{k}^{-0.5}+\mathrm{p}_{3} \mathrm{k}^{-0.5}-\mathrm{p}_{4} \mathrm{k}^{-0.5} \ldots \ldots$. Hence, Hückel cyclobutadiene has an orbital $0.5 p_{1}+0.5 p_{2}+0.5 p_{3}+0.5 p_{4}$ for which $r=-2$ and an orbital $0.5 p_{1}-0.5 p_{2}+0.5 p_{3}-0.5 p_{4}$ for which $r=2$.

Comparable simplicity attends symmetry-simplified calculations on Möbius annulenes with only one proviso: each basis set has a single pair of adjacent orbitals which are inverted 
(see $p_{2}, p_{3}$ at the top of Figure 9) - for this pair of orbitals an antibonding interaction requires their coefficients to have the same signs and a bonding interaction requires their coefficients to have opposite signs.
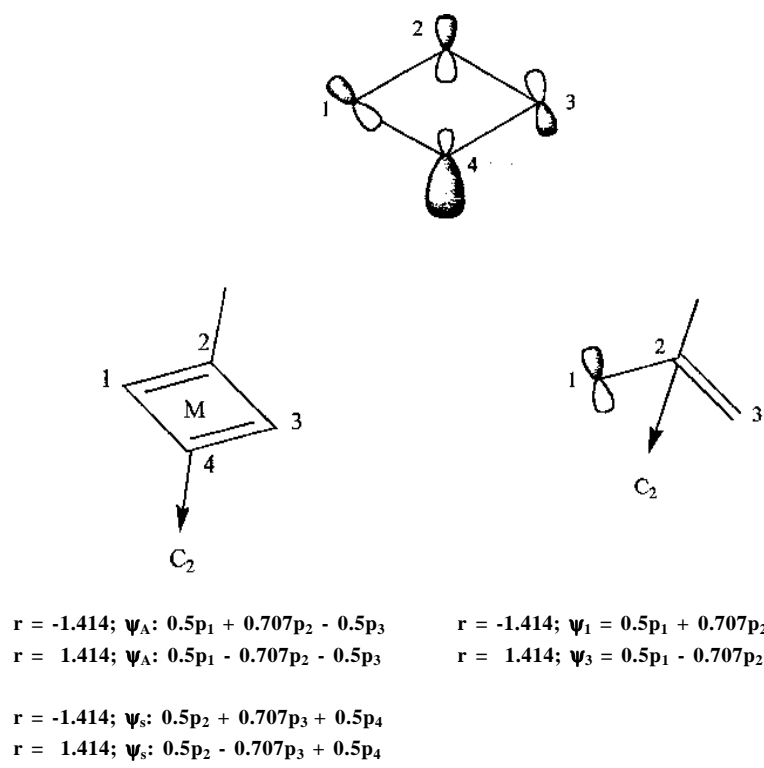

Figure 9. Simple access to degenerate eigenfunctions for Möbius cyclobutadiene.

Otherwise, half the molecular orbitals for Möbius annulenes are taken directly from those of the acyclic polyene with one carbon less. The homogeneous linear equations for Möbius cyclobutadiene are given below:

$\mathrm{a}_{1} \mathrm{r}+\mathrm{a}_{2}+0-\mathrm{a}_{4}=0$

$\mathrm{a}_{1}+\mathrm{a}_{2} \mathrm{r}+\mathrm{a}_{3}+0=0$

$0+\mathrm{a}_{2}+\mathrm{a}_{3} \mathrm{r}+\mathrm{a}_{4}=0$

$-\mathrm{a}_{1}+0+\mathrm{a}_{3}+\mathrm{a}_{4} \mathrm{r}=0$

Figure 9 shows the molecular orbitals for Möbius cyclobutadiene (see also Figure 5 for those roots which are common to the spectra of Möbius cyclobutadiene and the allyl group).

\section{CONCLUSIONS}

Hückel-level classroom lectures about aromaticity of Hückel and Möbius annulenes can be put on a quantitative basis without the need for lengthy computations. Hückel spectra may be obtained simply with the aid of the Frost or Zimmerman circle mnemonics and rudimentary trigonometry. Alternatively, the double circle mnemonic may be employed for most even Hückel annulene spectra in conjunction with rudimentary trigonometry.

Eigenfunctions and spectra for any acyclic polyene can be conveniently obtained with the Free Electron Method ${ }^{12}$. Thereafter, both spectra for an isoconjugate pair of Hückel and Möbius annulenes (with one more carbon) are immediately known except roots at \pm 2 . A simple generalization allows one to know when there are annulene roots at \pm 2 .

About half of the molecular orbitals for a Hückel or Möbius annulene are immediately available from those of the corresponding acyclic polyene with one less carbon. Remaining eigenfunctions are readily obtained by symmetry-simplified calculations using the appropriate homogemeous linear equations and the known secular equation roots. Results from the Langler semicircular mnemonic serve to caution against attempting to apply these methods or generalizations derived from them to non-annulenes.

\section{REFERENCES}

1. Huckel, E.; Z. Phys. 1931, 70, 204.

2. (a) Higasi, K.; Baba, H.; Rembaum, A.; Quantum Organic Chemistry, Interscience, New York, 1965; (b) Liberles, A.; Introduction to Molecular Orbital Theory, Holt, Rinehart and Winston, New York, 1966; (c) Yates, K.; Huckel Molecular Orbital Theory, Academic Press, New York, 1978.

3. (a) Dewar, M. J. S.; Angew. Chem. Int. Ed. Engl. 1971, 10, 761; (b) Zimmerman, H. E.; Acc. Chem. Res. 1971, 4, 272.

4. Langler, R. F.; J. Chem. Educ. 1996, 73, 899.

5. Trinajstic, N.; Chemical Graph Theory, Vols. 1 and 2, CRC Press, Boca Raton, Florida, 1983.

6. Moler, C.; MATLAB, University of New Mexico, 1981.

7. Heilbronner, E.; Tetrahedron Lett. 1964, 1923.

8. (a) Frost, A. A.; Musulin, B. J.; J. Phys. Chem. 1953, 21, 572; (b) Levine, I. N.; Quantum Chemistry, Prentice-Hall, Englewood Cliffs, N. J., 1991, pp. 558-560.

9. Salgado Moran, G.; Boletin Sociedad Quimica del Peru 1972, $45,117$.

10. Zimmerman, H. E.; J. Am. Chem. Soc. 1966, 88, 1564 and 1566.

11. Norinder, U.; Wennerstrom, O.; Wennerstrom, H.; Tetrahedron Lett. 1984, 25, 1397.

12. Langler, R. F.; Chem. Educator 1998, 3, S1430-4171(98) 021906.

13. Durkin, K. A.; Langler, R. F.; J. Phys. Chem. 1987, 91, 2422 and references therein. 